\title{
Jahrestagung 1998
}

Vom 7. bis 10. Oktober 1998 hielt die Vereinigung der Deutschen Staatsrechtslehrer in der 1991 gegründeten Universität Potsdam ihre Jahrestagung ab, an der einschließlich Begleitung und Gäste über 400 Personen teilnahmen - eine bisher nicht erreichte Zahl. Während der Mitgliederversammlung gedachte die Vereinigung ihrer seit der Osnabrücker Tagung verstorbenen Mitglieder Hans-Peter Ipsen, der seit 1989 Ehrenvorsitzender war, und Hartmut Krüger. Die Vereinigung wird das Andenken der Verstorbenen in Ehren halten. Der Vorsitzende hieß 23 neue Mitglieder willkommen, die sich kurz vorstellten. Damit zählt die Vereinigung jetzt 446 Mitglieder. Am ersten Abend fand im Schloß Bellevue, Berlin, ein Empfang durch den Bundespräsidenten, unser Mitglied Roman Herzog, statt, der am Donnerstag vormittag an den Beratungen teilnahm.

Als ersten Beratungsgegenstand hat der Vorstand zum wiederholten Male das parlamentarische Regierungssystem ausgewählt. Über Parlament und Regierung im modernen Staat sprachen 1957 Ernst Friesenhabn und Josef Partsch. 1974 hieß das Thema: „Das parlamentarische Regierungssystem des Grundgesetzes: Anlage - Erfahrungen - Zukunftseignung ${ }^{\alpha}$; die Berichte lieferten Thomas Oppermann und Hans Meyer. Nach 24 Jahren wurde nun erneut das parlamentarische Regierungssystem aufgegriffen, freilich unter dem vor den Septemberwahlen besonders aktuellen Aspekt des Bundesrates, dessen Zustimmungskompetenz sich im Laufe der Zeit immer mehr ausgeweitet hat. Außer dem aktuellen Anlaß ergab sich, daß 1998 für das Thema ein dreifaches Gedenkjahr ist: Vor 50 Jahren, im Herbst 1948, trat der Parlamentarische Rat zusammen, dem wir unser parlamentarisches Regierungssystem und den Bundesrat verdanken. Vor 80 Jahren, im Oktober 1918, wurde noch im Rahmen der Reichsverfassung von 1871 durch verfassungsänderndes Gesetz eine Parlamentarisierung der Reichsregierung versucht. Vor 150 Jahren, 1848, fanden in der Frankfurter Paulskirche die Verfassungsberatungen der Deutschen Nationalversammlung statt, die den Versuch unternommen hat, eine deutsche Verfassung auf demokratischem Wege zu etablieren.

Der zweite Beratungsgegenstand, Rechtliche Optimierungsgebote oder Rahmensetzungen für das Verwaltungshandeln" steht in deutlich erkennbarer Kontinuität zu früheren Beratungen über Verwaltungsverantwortung und Verwaltungsgerichtsbarkeit (1975), Verwaltungsverfahren und Verwaltungseffizienz (1982) und den Verwaltungsvorbehalt (1984). Es 
wurde erneut geprüft, wieweit das Gesetz das Verwaltungshandeln steuern kann und soll und - von der Gerichtsbarkeit aus gesehen - welches Ausmaß die Kontrolle der Verwaltung haben soll. Die Vorträge zum zweiten Beratungsgegenstand stellten insoweit eine Premiere dar, als nicht nur österreichisches und schweizerisches Recht sowie EG-Verwaltungsrecht vergleichend herangezogen wurden, sondern der dritte Berichterstatter sich auf Anregung des Vorstandes der Thematik vom englischen und französischen Recht her zuwandte.

Der Gesprächskreis Verwaltungslehre tagte am Mittwoch, dem 7. Oktober 1998. Die Herren F. Kirchhof und Trzaskalik referierten über haushalts- und staatsschuldenrechtliche Probleme neuer Finanzierungsmodelle im Bereich der öffentlichen Verwaltung. Zum neuen Vorsitzenden des Gesprächskreises wurde Herr Bull gewählt.

Eine Gruppe von Mitgliedern traf sich am selben Tag zur Bildung eines Arbeitskreises „Europäisches Verfassungsrecht ${ }^{\text {. }}$. Herr $E$. Klein wurde zum Vorsitzenden, Herr Pernice zu seinem Stellvertreter gewählt. Die Mitgliederversammlung bestätigte einstimmig die Gründung dieses Arbeitskreises, der in Zukunft gleichzeitig mit dem Arbeitskreis „Verwaltungslehre“ am Mittwoch vor der Mitgliederversammlung tagen wird. Der Arbeitskreis soll inhaltlich auf drei Problemkreise ausgerichtet sein:

- die Rechtsvergleichung mit Schwerpunkt im europäischen Bereich,

- die Entwicklung von Standards eines gemeineuropäischen Verfassungsrechts

und

- die Untersuchung der Wechselwirkungen zwischen den einzelnen nationalen öffentlich-rechtlichen Systemen und dem europäischen Recht.

Am Donnerstag abend fand ein Empfang durch den Ministerpräsidenten des Landes Brandenburg, Herrn Dr. Manfred Stolpe, und den Rektor der Universität Potsdam, unser Mitglied Wolfgang Loschelder, in den Räumen des Hauptgebäudes der Juristischen Fakultät und der Wirtschafts- und Sozialwissenschaftlichen Fakultät statt. Der gesellige Abend am Freitag stimmte durch eine Fontane-Lesung die Teilnehmer ein auf den Ausflug am Samstag in die Fontane-Stadt Neuruppin und nach Wustrau.

Die Vereinigung schuldet vor allem Michael Nierhaus, dem Dekan der Fakultät und kooptierten Vorstandsmitglied, und seinen Mitarbeitern tiefen Dank für die vorzügliche Vorbereitung und die höchsten Standards der Verwaltungspraxis genügende Organisation der Tagung. Frau Klein und Frau Loschelder haben mit dem interessanten Begleitprogramm den Teilnehmern die Baulichkeiten und die Geschichte Potsdams erschlossen, wofür ihnen der herzliche Dank der Vereinigung gilt.

C.S. 